\title{
Correlation between ultrasound Diagnosis of Chorionicity with postpartum placental Histopathology and the Risk of adverse perinatal Outcomes in monochorionic twin Pregnancies
}

\footnotetext{
Authors: $\quad$ Silvia Juliana Barajas Rueda, Carlos Hernan Becerra

Submitted: 20. February 2020

Published:

Volume:

Issue:

Affiliation:

Languages:

Keywords:

Categories:

DOI:

22. February 2020

7

1

Abstract:

Universidad Industrial de Santander, Colombia.

Spanish, Castilian

Chorionicity, monochorionic, correlation, twin.

Medicine

10.17160/josha.7.1.632

The objective of this study is to determine if non-correlation between the ultrasound diagnosis of chorionicity with a postpartum placental histopathological study is a risk factor for presenting adverse perinatal outcomes, assumed fact, but it has not been registered yet in the current literature. MATERIALS AND METHODS: transversal and retrolective study. Twin pregnancies cases treated in the HUS between 2014-2019 were evaluated. Cases with a postpartum placental histopathological study report, concerning monochorionic twin pregnancy, were included. The ultrasound / histopathological diagnostic correlation of chorionicity was evaluated between initial obstetric ultrasound and placental study. Stratified sampling analysis was performed according to the presence or absence of diagnostic correlation and according to the time of the obstetric ultrasound.
}

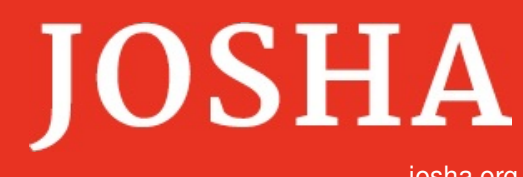

josha.org
Journal of Science, Humanities and Arts

JOSHA is a service that helps scholars, researchers, and students discover, use, and build upon a wide range of content 


\section{Correlation between ultrasound diagnosis of chorionicity with postpartum placental histopathological study and its relationship with the risk to present adverse perinatal outcomes in monochorionic twin pregnancies}

CORRELACIÓN ENTRE EL DIAGNÓSTICO ECOGRÁFICO DE LA CORIONICIDAD CON EL ESTUDIO HISTOPATOLÓGICO PLACENTARIO POST PARTO Y SU RELACIÓN CON EL RIESGO PARA PRESENTAR DESENLACES PERINATALES ADVERSOS EN EMBARAZOS GEMELARES MONOCORIALES

Silvia Juliana Barajas Rueda1, Carlos Hernán Becerra2

1. Especialista en ginecología y obstetricia. Hospital Universitario de Santander, Universidad Industrial de Santander. Bucaramanga, Santander, Colombia. Correo: sjbr_25@hotmail.com

2. Medico Ginecólogo-Obstetra, Especialista en Medicina Materno Fetal, Profesor Departamento de Ginecología y Obstetricia. Escuela de Medicina, Facultad de Salud. Universidad Industrial de Santander, Bucaramanga, Colombia.

Autor principal y de correspondencia: Silvia Juliana Barajas Rueda. 


\section{ABSTRACT}

OBJECTIVE: To determine if non-correlation between the ultrasound diagnosis of chorionicity with a postpartum placental histopathological study is a risk factor for presenting adverse perinatal outcomes, assumed fact, but it has not been registered yet in the current literature.

MATERIALS AND METHODS: transversal and retrolective study. Twin pregnancies cases treated in the HUS between 2014-2019 were evaluated. Cases with a postpartum placental histopathological study report, concerning monochorionic twin pregnancy, were included. The ultrasound / histopathological diagnostic correlation of chorionicity was evaluated between initial obstetric ultrasound and placental study. Stratified sampling analysis was performed according to the presence or absence of diagnostic correlation and according to the time of the obstetric ultrasound.

RESULTS: 202 twin pregnancies cases were evaluated; 47\% were monochorionic. No diagnostic correlation of chorionicity was found in $22 \%$ of cases. The prevalence of overall adverse perinatal outcome was 71\% with RP 3 CI 95\% (17.9 - 8.0) and the prevalence of neonatal complication was $100 \%$ with RP 7 CI 95\% (1.9 - 25.2) in the subgroup that presented no diagnostic correlation and its initial ultrasound was taken after 20 weeks of gestation.

CONCLUSION: According to results, it was determined that the non-correlation between the ultrasound / histopathological diagnosis of chorionicity, in ultrasound scans taken late, increases the risk of presenting neonatal complications 7 times, and 3 times the risk of presenting overall adverse perinatal outcome in monochorionic twin pregnancies; assumed fact, but not established statistically until the moment of this study.

KEYWORDS: Chorionicity, monochorionic, correlation, twin. 


\section{RESUMEN}

OBJETIVO: Determinar si la no correlación entre el diagnóstico ecográfico de la corionicidad con el estudio histopatológico placentario postparto es un factor de riesgo para presentar desenlaces perinatales adversos, hecho supuesto, pero aún no demostrado ni descrito en la literatura actual.

MATERIALES Y METODOS: Estudio trasversal, retrolectivo. Se evaluaron casos con embarazo gemelar, atendidos en el HUS, entre 2014-2019. Se incluyeron, los casos con reporte de estudio patológico placentario post parto, con referencia de embarazo gemelar monocorial. Se evalúo, la correlación diagnostica ecográfica/histopatológica de la corionicidad, entre la ecografía obstétrica inicial y el estudio de la placenta. Se realizó análisis por estratos según presencia o no de correlación diagnóstica y según momento de toma de ecografía obstétrica.

RESULTADOS: Se evaluaron 202 casos de embarazos gemelares; $47 \%$ fueron monocoriales. Se encontró no correlación diagnóstica de la corionicidad en $22 \%$ de los casos. La prevalencia de resultado perinatal adverso global fue de 71\% con RP 3 IC 95\% (17.98.0) y la prevalencia de complicación neonatal fue de $100 \%$ con RP de 7 IC $95 \%$ (1.9 - 25.2) en el subgrupo que presento no correlación diagnostica y su ecografía inicial fue tomada después de las 20 semanas de gestación.

CONCLUSION: Según los resultados, se determinó que la no correlación entre el diagnóstico ecográfico/histopatológico de la corionicidad, en ecografías tomadas tardíamente, aumenta 7 veces el riesgo de presentar complicaciones neonatales, y 3 veces le riesgo de presentar resultado perinatal adverso global en embarazos gemelares monocoriales; hecho supuesto, pero no establecido estadísticamente hasta el momento de este estudio.

Palabras clave: corionicidad, monocorial, correlación, gemelar. 


\section{INTRODUCCION}

Los embarazos gemelares corresponden a 1 de cada 80 embarazos; están asociados a mayores tasas de complicaciones, contribuyen con una mortalidad perinatal 3 a 5 veces mayor que en embarazos simples y tienen mayor morbilidad que los embarazos únicos, con 10 veces más riesgo de lesiones neurológicas fetales(1). Es de anotar además que lo embarazos monocoriales presentan mayor tasa de complicaciones y mayor morbi-mortalidad perinatal comparado con los embarazos gemelares bicoriales. La mortalidad en los embarazos monocoriales se subestima si se tiene en cuenta que gran parte de las perdidas ocurren antes de la semana 24 de gestación(2).

Una tercera parte de los embarazos gemelares son monocigóticos, y de estos el 70.0\% son monocoriales, es decir comparten una placenta; estos están en riesgo de presentar complicaciones exclusivas derivadas del hecho de ser monocoriales, tales como : síndrome de transfusión de gemelo a gemelo, RCIU selectivo, feto acardio, entrecruzamiento de cordones (este es exclusivo de embarazos gemelares monocoriales monoamnióticos) que pueden ser letales o asociarse con morbilidad grave (3).

Derivado de las observaciones anteriores se ha planteado un seguimiento diferencial para los embarazos gemelares monocoriales de los bicoriales, no solo en la frecuencia de su seguimiento, sino también en la edad gestacional de finalización del embarazo; para ello se ha establecido que la ecografía es el único método seguro y fiable para el diagnóstico prenatal de la corionicidad en el embarazo gemelar. La evaluación ecográfica temprana proporciona una estimación precisa de la edad gestacional además de determinar la corionicidad $(4,5)$.Esto es crítico porque los gemelos monocoriales tienen una circulación feto-placentaria compartida, lo que los pone en riesgo de complicaciones específicas graves del embarazo ya expuestas anteriormente.

A pesar de ser la ecografía el método más confiable y seguro para el diagnóstico de la corionicidad en el embarazo gemelar, se debe recordar que su exactitud en el diagnostico 
depende de muchos factores, tales como la experiencia del operador y el trimestre en el que se realiza el diagnóstico $(3,6)$.

Si bien, el determinar el tipo de corionicidad de forma oportuna y exacta, parece tener sentido lógico para predecir y manejar complicaciones de forma temprana y así disminuir la morbimortalidad fetal, aún no se ha evaluado el hecho de si hacerlo o no implica presentar mayor riesgo de complicaciones; tomando en cuenta lo anterior este proyecto busca determinar si la no concordancia entre el diagnóstico ecográfico de la corionicidad con el estudio histopatológico placentario postparto es un factor de riesgo para presentar desenlaces perinatales adversos(relacionados con la corionicidad), hecho supuesto pero aún no demostrado ni descrito en la literatura actual.

\section{MATERIALES Y METODOS}

Se realizo un estudio trasversal retrolectivo, que incluyo gestantes cuyo parto fue atendido en el Hospital Universitario de Santander entre los años 2014-2019, y que cumplieron los siguientes criterios: ser embarazo gemelar, tener al menos un reporte ecográfico con descripción del tipo de corionicidad presentada, sin importar el sitio de realización de esta ni la edad gestacional del informe más temprano y tener reporte de estudio postparto de la placenta con descripción de tipo de corionicidad.

La búsqueda de los casos se realizo en el sistema de ingresos del departamento de sala de partos, del hospital universitario de Santander, a partir de la identificación de los términos “embarazo múltiple” y “embarazo gemelar”; adicionalmente, se complementó con la revisión manual del libro de ingresos del mismo departamento.

En todos los casos incluidos, se revisó el diagnóstico de la corionicidad según las ecografías realizadas en su control prenatal y se comparó con el informe de patología, siendo el factor de riesgo estudiado la no correlación entre el diagnóstico ecográfico de la corionicidad y el estudio histopatológico placentario postparto. 
Las variables estudiadas fueron: edad materna, procedencia, escolaridad, causa de finalización de la gestación, número de controles pręnatales, edad gestacional de toma de la primera ecografía obstétrica, presencia de complicación fetal, complicación neonatal, muerte fetal, muerte neonatal y muerte perinatal.

Se realizo análisis estratificado, inicialmente en dos grupos, según correlación diagnóstica, aquí, se presentan las características demográficas y clínicas entre los dos grupos de comparación. Las diferencias en las proporciones se estimaron según la prueba estadística de chi cuadrado o prueba exacta de Fischer según correspondiera con los supuestos estadísticos y se calculó la razón de prevalencia para cada uno. Posteriormente se realizó el análisis organizando los casos de no concordancia diagnóstica de la corionicidad según la edad gestacional al momento de realizar la ultrasonografía, conformándose dos subgrupos (ecografía diagnóstica de corionicidad antes o en la semana 20 de gestación y ecografía diagnóstica realizada después de las 20 semanas) tomando el estudio histopatológico placentario post-parto en embarazos gemelares como la prueba de referencia para establecer la corionicidad.

Se organizaron las variables desenlaces motivo de estudio como resultado perinatal fatal (corresponden a muerte fetal o muerte perinatal y resultado perinatal adverso global (compuesto que incluye mortalidad y/o morbilidad perinatal). Para el análisis bivariado se conformaron variables como edad categorizada, procedencia categorizada, escolaridad categorizada.

Se estableció la razón de prevalencia de las diversas complicaciones fetales y neonatales derivadas de la corionicidad tanto en los casos de no concordancia diagnóstica ecográficapatológica de la corionicidad, tanto en la población de embarazos monocoriales como en los dos subgrupos definidos anteriormente. 
Aspectos éticos: El proyecto fue aprobado por el comité de ética de la Universidad Industrial de Santander, así como también fue aprobado por el comité de ética del Hospital Universitario de Santander.

\section{RESULTADOS}

En total se evaluaron 202 casos de embarazos gemelares atendidos en el Hospital Universitario de Santander entre los años 2014-2019; de estos 94 casos (47\%) corresponde a embarazos gemelares monocoriales y 108 casos (53\%) fueron embarazos gemelares bicoriales biamnióticos. Se presentan a continuación los resultados correspondientes a los embarazos gemelares monocoriales, objetivo de este estudio. Se evaluaron tanto variables fetales como neonatales, con la no correlación diagnóstica ecográfico/histopatológica en los embarazos gemelares monocoriales; no se obtuvo información respecto a variables neonatales en 13 casos (14\%).

En el grupo de pacientes con embarazo gemelar monocorial, las gestantes tenían edad entre los 18-41 años, la mediana de edad fue 28 años (RIQ 23-33).

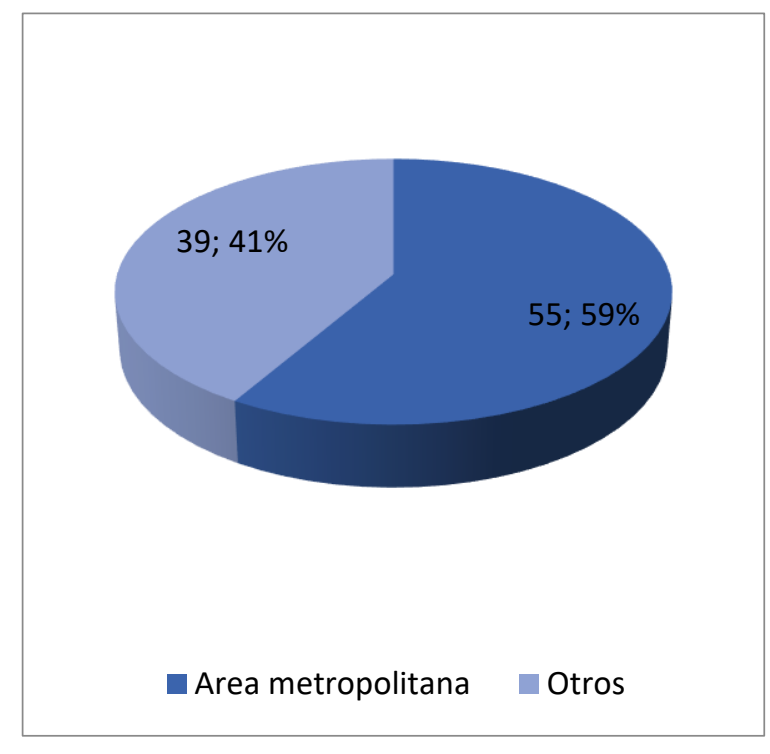

Figura 1 Distribución demográfica embarazos gemelar monocorial HUS

2014-2019

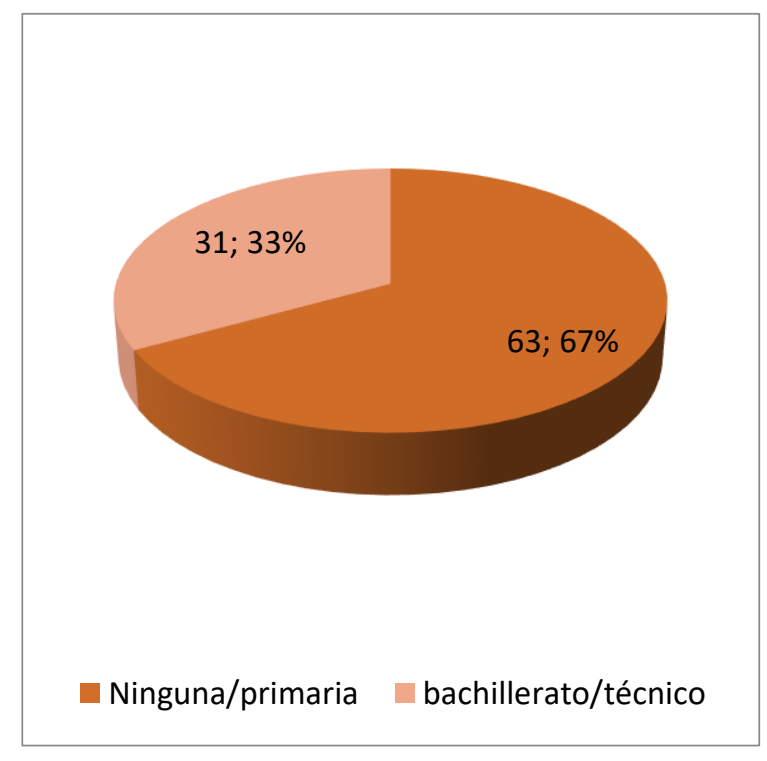

Figura 2 Nivel de escolaridad en Pacientes con embarazo gemelar monocorial HUS 2014-2019 
La mayoría de los casos, 55 (59\%) provenían del área metropolitana: Bucaramanga, Floridablanca, Girón y Piedecuesta y 39 casos (41\%) de otros sitios del país. Ver figura 2; y cerca de 63 casos, (67\%) tenían básica primaria o no estaban escolarizadas.

El 50\% de los casos de embarazos gemelares monocoriales contaban con más de 3 controles prenatales y solo (18) 19 \% casos no tenía ningún control prenatal, figura 3; además se encontró que 67 casos $(71 \%)$ contaban con ecografía obstétrica antes de la semana 20 de gestación que describía el tipo de corionicidad y solo en 27 (29\%) casos se realizó después de semana 20.

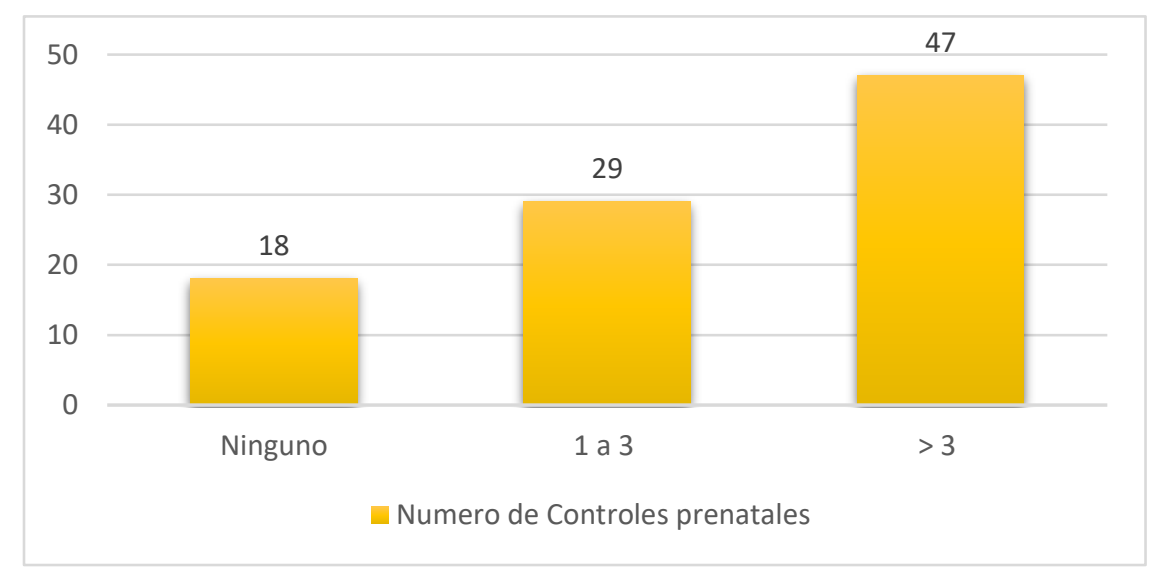

Figura 3 Número de Controles prenatales en paciente con embarazo gemelar monocorial HUS 2014-2019

La media de edad gestacional de terminación de la gestación fue de 34.3 (RIQ 31.5 - 36.5) semanas. Entre las causas de finalización de la gestación, se encontró que 38 casos (40\%) fue trabajo de parto pretérmino, 22 casos (23\%) cesárea programada; trastornos hipertensivos (8\%) para 7 casos, RPM 6 casos (7\%), aborto 6 casos ( $6 \%$ ) uno de ellos correspondiente a IVE (siameses dicéfalos - parápagos) e infección intra-amniótica 2 casos (2\%); además 13 casos (14\%) correspondieron a aquellas causas relacionadas con complicaciones derivadas de la corionicidad; principalmente alteración Doppler fetal en casos con diagnóstico de RCIU o Síndrome de trasfusión feto fetal. 


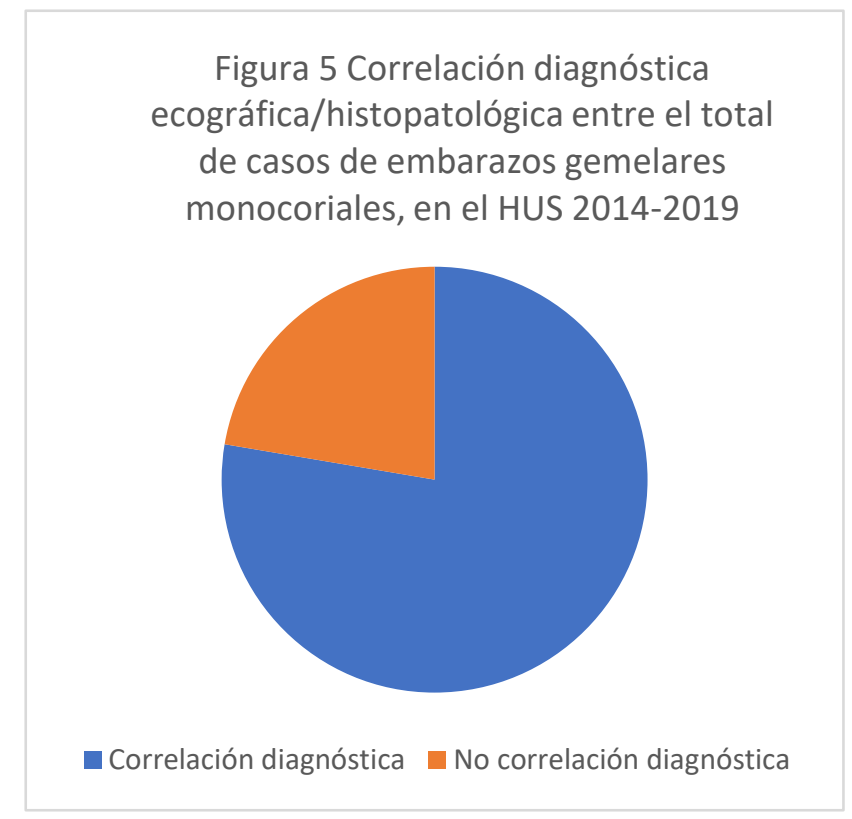

En relación con la no correlación diagnóstica de la corionicidad entre ecografía/histopatología, encontramos no correlación en 21 casos (22\%). Ver figura 5 .

Dentro de nuestra población estudiada, no se encontró diferencias significativas entre edad materna, procedencia ni nivel de escolaridad, con la presentación de complicaciones perinatales, tanto en el grupo de no correlación diagnóstica de la corionicidad como en el grupo que presentaban dicha correlación; independiente del momento de toma de la ecografía, ver tabla 2 y 3.

\begin{tabular}{|c|c|c|c|c|}
\hline & $\begin{array}{l}\text { No correlación diagnóstica de } \\
\text { corionicidad } \\
\text { ecográfico/histopatológico } \\
\text { (N 21) (\%) }\end{array}$ & $\begin{array}{l}\text { Correlación diagnóstica de } \\
\text { corionicidad } \\
\text { Ecográfico/histopatológico } \\
\qquad(\mathrm{N}=73)(\%)\end{array}$ & $\begin{array}{c}\text { Prueba } \\
\text { exacta de } \\
\text { Fischer } * \\
\text { Chi } \\
\text { cuadrado } \\
\text { (p) }{ }^{\circ}\end{array}$ & $\begin{array}{c}\text { Razón de } \\
\text { Prevalencia } \\
\text { IC95\% }\end{array}$ \\
\hline $\begin{array}{l}\text { Edad materna } \\
\qquad \begin{array}{l}\text { Menor } 30 \text { años } \\
\text { Mayor } 30 \text { años }\end{array}\end{array}$ & $\begin{array}{l}12(57) \\
9(43)\end{array}$ & $\begin{array}{l}47(64) \\
26(36)\end{array}$ & $0.545^{\circ}$ & $1.2(0.6-2.1)$ \\
\hline $\begin{array}{l}\text { Procedencia } \\
\text { Área metropolitana } \\
\text { Otros }\end{array}$ & $\begin{array}{l}13(62) \\
8(38)\end{array}$ & $\begin{array}{l}42(58) \\
31(43)\end{array}$ & $0.720^{\circ}$ & $1.0(1-1)$ \\
\hline $\begin{array}{l}\text { Escolaridad } \\
\text { Ninguno/Primaria } \\
\text { Bachillerato/Técnico }\end{array}$ & $\begin{array}{l}17(81) \\
4(19)\end{array}$ & $\begin{array}{l}46(63) \\
27(37)\end{array}$ & $0.123^{\circ}$ & $0.5(0.2-1.3)$ \\
\hline
\end{tabular}

Tabla 2. Caracterización según correlación diagnóstica ecográfica/histopatológico de los embarazos gemelares monocoriales, HUS 2014-2019 
Tabla 3. Caracterización de los embarazos gemelares según edad gestacional de toma de ecografía en pacientes con no correlación diagnóstica ecográfica/histopatológico, HUS

2014-2019

\begin{tabular}{|c|c|c|c|c|}
\hline & $\begin{array}{c}\text { Ecografía mayor de } \\
\text { semana } 20 \text { de gestación } \\
(\mathrm{N}: 7)(\%)\end{array}$ & $\begin{array}{c}\text { Ecografía menor de } \\
\text { semana } 20 \text { de gestación } \\
(\mathrm{N}: 14)(\%)\end{array}$ & $\begin{array}{l}\text { Prueba exacta de } \\
\text { Fischer* } \\
\text { Chi cuadrado }{ }^{\circ} \\
\text { (p) }\end{array}$ & $\begin{array}{c}\text { Razón de } \\
\text { Prevalencia } \\
\text { IC95\% }\end{array}$ \\
\hline $\begin{array}{l}\text { Procedencia } \\
\text { Área metropolitana } \\
\text { Otros }\end{array}$ & $\begin{array}{l}4(57) \\
3(43)\end{array}$ & $\begin{array}{l}9(64) \\
5(36)\end{array}$ & $0.557^{*}$ & $1(1-1)$ \\
\hline $\begin{array}{l}\text { Escolaridad } \\
\text { Ninguno/Primaria } \\
\text { Bachillerato/Técnico }\end{array}$ & $\begin{array}{l}7(100) \\
0(0)\end{array}$ & $\begin{array}{l}10(71) \\
4(29)\end{array}$ & $0.255 *$ & $* * * * * *$ \\
\hline
\end{tabular}

Se encontró una prevalencia de complicaciones fetales derivadas de la corionicidad en el total de casos de embarazos gemelares monocoriales fue de 31 casos (33\%), dentro de las cuales encontramos: 18 casos $(55 \%)$ correspondieron a RCIU, 9 casos (27\%) a Síndrome de trasfusión feto-fetal, entrecruzamiento de cordón 1 caso (3\%), feto acárdico correspondiente a 1 caso (3\%); y adicional encontramos 2 casos (6\%) de fetos siameses (dicéfalos-parápagos y dicefalos-toraco-onfalopagos).

En relación a la mortalidad fetal encontrada, tenemos 13 (14\%) casos; de estos, 6 casos fueron abortos, definido como perdida gestacional antes de las 22 semanas de gestación, correspondiendo a un $46 \%$ y 7 casos correspondieron a muertes fetales tardías, es decir aquellos muertes fetales in útero que ocurren entre semana 22 hasta el término de la gestación, correspondiendo a un (54\%); entre estos tenemos: 3 casos (43\%) con síndrome de trasfusión feto-fetal, en quienes se realizó fotocoagulación laser entre semanas 18 y 19 en dos casos; y cuya causa de finalización de gestación fue obstétrica: trabajo de parto pretérmino y ruptura de membranas, los tres casos con un feto obitado; un caso (14\%) con RCIU y finalización de gestación por trabajo de parto pretérmino, 3 casos (43\%) no relacionados con complicaciones derivadas de la corionicidad y cuya finalización fue de causa obstétrica ( trabajo de parto pretérmino ) con feto obitado . Además, se encontró dentro 
del subgrupo que presento muerte fetal temprana, un caso (17\%) de siameses dicefalosparapagos con indicación de IVE, el resto de los casos correspondieron a abortos espontáneos

La prevalencia de complicaciones neonatales, en el total de casos de embarazo gemelar monocorial, fue de 33 (41\%) casos; se consideraron para el presente estudio las siguientes: SDR 33 casos (41\%), hemorragia interventricular 2 casos (2.5\%), sepsis 5 casos (6\%), anemia neonatal 6 casos (7\%) y necesidad de trasfusión 2 casos $(2.5 \%)$, ingreso a Unidad de cuidado intensivo neonatal (12 casos (15\%); recordando que no se obtuvo información respecto a 13 casos $(14 \%)$.

Se identificaron 7 casos $(8 \%)$ de muerte neonatal, además de 11 casos (14\%) correspondientes a muerte perinatal, entendida esta como la presencia de muerte fetal tardía y/o muerte neonatal temprana, en el total de la población estudiada. Las muertes neonatales encontradas correspondieron a : 3 casos (43\%) presentaron complicación fetal derivada de la corionicidad ( TFF) y cuya causa de finalización del embarazo del total de casos, fue trabajo de pato pretérmino, 3 (43\%) casos no relacionados con la presencia de complicación fetal derivada de la corionicidad y cuya causa de finalización de la gestación fue trabajo de parto pretérmino en embarazos <24 semanas de gestación; además se encontró un caso (14\%) de siameses con diagnóstico tardío de dicha condición y cuya causa de finalización fue parto pre término (paciente sin control prenatal).

Se determinó, en el grupo de casos con embarazo gemelar monocorial que presentaron no correlación diagnostica ecográfico/histopatológica de la corionicidad, comparado con el grupo de casos que si la presentaron: la prevalencia de resultado perinatal adverso global (estableciendo este como la presencia de mortalidad perinatal y/o complicaciones neonatales) y de Riesgo perinatal adverso fatal (mortalidad perinatal) en embarazos monocoriales, fue de $10 \%$ con RP 0.8 IC 95\% (0.1 - 3.7) y 16\% con RP 1.2 IC 95\% (0.3 - 4.1) respectivamente, siendo no significativo estadísticamente; a su vez no se encontró relación estadística significativa entre la presencia de complicaciones fetales derivadas de la corionicidad, cuya 
prevalencia fue de $14 \%$ con RP 0.3 IC $95 \%(0.1-1.1)$; muerte fetal con prevalencia de 14\%, con RP 1.0 IC $95 \%$ (0.3 - 3.4), complicación neonatal con prevalencia de $38 \%$ con RP 0.8 IC 95\% (0.4 - 1.5), muerte neonatal de $11 \%$ con RP 1.3 IC 95\% (0.2 - 6.1) y muerte perinatal con prevalencia de $16 \%$ con RP 1.2 IC $95 \%$ (0.3-4.1), en este grupo de pacientes. Ver tabla 4

Tabla 4. Complicaciones fetales y neonatales según concordancia diagnóstica ecográfica/histopatológico en embarazos gemelares monocoriales, HUS 2014-2019

\begin{tabular}{|c|c|c|c|c|}
\hline & $\begin{array}{l}\text { No correlación diagnostica } \\
\text { de corionicidad } \\
\text { ecográfico/histopatológico } \\
\qquad \mathrm{N}=21(\%)\end{array}$ & $\begin{array}{l}\text { Correlación diagnostica } \\
\text { de corionicidad } \\
\text { Ecográfico/histopatológico } \\
\qquad \mathrm{N}=73(\%)\end{array}$ & $\begin{array}{c}\text { Prueba } \\
\text { exacta de } \\
\text { Fischer } \\
\text { Chi } \\
\text { cuadrado }\end{array}$ & $\begin{array}{c}\text { Razón de } \\
\text { prevalencia } \\
\text { IC95\% }\end{array}$ \\
\hline $\begin{array}{l}\text { Complicación fetal } \\
\mathrm{Si} \\
\text { No }\end{array}$ & $\begin{array}{l}3(14) \\
18(86)\end{array}$ & $\begin{array}{l}28(38) \\
45(62)\end{array}$ & $\mathbf{0 . 0 3 9}^{\circ}$ & $.3(0.1-1.1)$ \\
\hline $\begin{array}{l}\text { Complicación } \\
\text { Neonatal } \\
\mathrm{Si} \\
\mathrm{No}\end{array}$ & $\begin{array}{l}8(38) \\
13(62)\end{array}$ & $\begin{array}{l}32(44) \\
41(56)\end{array}$ & $0.639^{\circ}$ & $0.8(0.4-1.5)$ \\
\hline $\begin{array}{l}\text { Muerte fetal } \\
\mathrm{Si} \\
\text { no }\end{array}$ & $\begin{array}{l}3(14) \\
18(86)\end{array}$ & $\begin{array}{l}10(14) \\
63(86)\end{array}$ & $0.945^{\circ}$ & $1.0(0.3-3.4)$ \\
\hline $\begin{array}{l}\text { Muerte Neonatal } \\
\mathrm{Si} \\
\text { No }\end{array}$ & $\begin{array}{l}2(11) \\
17(89)\end{array}$ & $\begin{array}{l}5(8) \\
57(92)\end{array}$ & $0.738 *$ & $1.3(0.2-6.1)$ \\
\hline $\begin{array}{l}\text { Muerte perinatal } \\
\mathrm{Si} \\
\text { No }\end{array}$ & $\begin{array}{l}3(16) \\
16(84)\end{array}$ & $\begin{array}{l}8(13) \\
54(87)\end{array}$ & $0.714^{\circ}$ & $1.2(0.3-4.1)$ \\
\hline $\begin{array}{l}\text { Riesgo perinatal } \\
\text { adverso global } \\
\mathrm{Si} \\
\text { No }\end{array}$ & $\begin{array}{l}2(10) \\
19(90)\end{array}$ & $\begin{array}{l}8(11) \\
65(89)\end{array}$ & $0.606^{*}$ & $0.8(0.1-3.7)$ \\
\hline $\begin{array}{l}\text { Riesgo perinatal } \\
\text { adverso fatal }\end{array}$ & & & & \\
\hline
\end{tabular}




\begin{tabular}{|l|l|l|l|l|}
\hline Si & $3(16)$ & $8(13)$ & $0.748^{*}$ & $1.2(0.3-4.1)$ \\
\hline No & $16(84)$ & $54(87)$ & & \\
\hline
\end{tabular}

Sin embargo, al analizar el grupo de casos de embarazo gemelar que no presentaron correlación diagnostica ecográfica/ histopatológica de la corionicidad, según momento de toma de la ecografía obstétrica inicial (antes de semana 20 vs después de semana 20), se encontró que el tener no correlación diagnóstica ecográfico/histopatológico de la corionicidad en ecografía mayor de 20 semanas, aumenta el riesgo de presentar resultado perinatal adverso global, RP 3 IC 95\% (17.9 - 8.0) con una prevalencia encontrada de $71 \%$ ; relación no evidente en el riesgo de presentar resultado perinatal adverso fatal, en este subgrupo de pacientes, ver tabla 5. Además, en este subgrupo se presentaron 5 casos (100\%) con complicación neonatal, los cuales tenían ecografía tomada después de semana 20, siendo esta relación significativa, con una RP de 7 IC 95\% (1.9 - 25.2) como se muestra en la tabla 5 .

Tabla 5. Complicaciones fetales y neonatales en casos de no correlación diagnóstica ecográfica/histopatológico y edad gestacional de toma de ecografía en embarazos gemelares monocoriales, HUS 2014-2019

\begin{tabular}{|c|c|c|c|c|}
\hline & $\begin{array}{l}\text { Ecografía mayor de } \\
\text { semana } 20 \text { de gestación } \\
\mathrm{N}=7(\%)\end{array}$ & $\begin{array}{l}\text { Ecografía menor de } \\
\text { semana } 20 \text { de gestación } \\
\mathrm{N}=14(\%)\end{array}$ & $\begin{array}{l}\text { Prueba exacta de } \\
\text { Fischer* } \\
\text { Chi cuadrado }^{\circ} \\
\text { (P) }\end{array}$ & $\begin{array}{l}\text { Razón de } \\
\text { prevalencia } \\
\text { IC95\% }\end{array}$ \\
\hline $\begin{array}{l}\text { Complicación fetal } \\
\mathrm{Si} \\
\text { No }\end{array}$ & $\begin{array}{l}1(14) \\
6(86)\end{array}$ & $\begin{array}{l}2(14) \\
12(86)\end{array}$ & $0.726 *$ & $1(0.1-9.2)$ \\
\hline $\begin{array}{l}\text { Complicación } \\
\text { neonatal } \\
\mathrm{Si} \\
\text { No }\end{array}$ & $\begin{array}{l}5(100) \\
0(0)\end{array}$ & $\begin{array}{l}2(14) \\
12(86)\end{array}$ & $0.0006 *$ & $7(1.9-25.2)$ \\
\hline $\begin{array}{l}\text { Muerte fetal } \\
\mathrm{Si} \\
\text { No }\end{array}$ & $\begin{array}{l}2(29) \\
5(71)\end{array}$ & $\begin{array}{l}1(7) \\
13(93)\end{array}$ & $0.186^{\circ}$ & $4(0.4-36.9)$ \\
\hline
\end{tabular}




\begin{tabular}{|c|c|c|c|c|}
\hline $\begin{array}{l}\text { Muerte Neonatal (2) } \\
\mathrm{Si} \\
\text { No }\end{array}$ & $\begin{array}{l}2(40) \\
3(60)\end{array}$ & $\begin{array}{l}0(0) \\
14(100)\end{array}$ & $0.058 *$ & $* * * *$ \\
\hline $\begin{array}{l}\text { Muerte perinatal } \\
\mathrm{Si} \\
\text { No }\end{array}$ & $\begin{array}{l}2(40) \\
3(60)\end{array}$ & $\begin{array}{l}1(7) \\
13(93)\end{array}$ & $0.155^{*}$ & $5.6(0.6-49.1)$ \\
\hline $\begin{array}{l}\text { Rperinatal adverso } \\
\text { global } \\
\mathrm{Si} \\
\text { No }\end{array}$ & $\begin{array}{l}2(29) \\
5(71)\end{array}$ & $\begin{array}{l}0(0) \\
14(100)\end{array}$ & $0.100 *$ & $3.8(1.7-8.0)$ \\
\hline $\begin{array}{l}\text { Rperinatal adverso } \\
\text { fatal } \\
\mathrm{Si} \\
\mathrm{No}\end{array}$ & $\begin{array}{l}2(40) \\
3(60)\end{array}$ & $\begin{array}{l}1(7) \\
13(93)\end{array}$ & $0.155^{*}$ & $5.6(0.6-49.1)$ \\
\hline
\end{tabular}

No se encontró relación estadística significativa entre el momento de la toma de la ecografía obstétrica inicial y la presencia de complicación fetal con una RP 1 IC $95 \%$ (0.1 - 9.2), muerte fetal, RP 4 IC 95\% (0.4 - 36.9), muerte perinatal RP 5.6 IC 95\% (0.6 - 49.1) ni muerte neonatal, en este subgrupo de pacientes, tal como se muestra en la tabla 5.

\section{DISCUSIÓN}

Se ha propuesto que el diagnóstico oportuno y preciso de la corionicidad en el embarazo gemelar, específicamente en el embarazo monocorial, disminuye el riesgo de presentar desenlaces perinatales y adversos, por lo tanto, se ha especificado que el I trimestre es el momento propicio para su realización, dado que hay reportes en la literatura mundial de alta sensibilidad y especificidad. $(2,7,13)$

Encontramos una prevalencia de no correlación diagnostica de la corionicidad del 22\%, dato un poco más bajo que el encontrado en la literatura (1). 
No se encontró relación estadísticamente significativa entre la no correlación en el diagnóstico ecográfico/histopatológico con el riesgo de presentar complicación neonatal, resultado perinatal adverso global ni en resultado perinatal adverso fatal.

Sin embargo, al analizar dicha relación en pacientes con no correlación diagnostica dependiendo de la edad gestacional de toma de la ecografía inicial, se encontró que el presentar no correlación diagnostica ecográfica/histopatológica de la corionicidad en ecografía tomada después de la semana 20 de gestación, aumenta 7 veces el riesgo de presentar complicaciones neonatales, y 3 veces le riesgo de presentar resultado perinatal adverso global en embarazos gemelares monocoriales; lo cual evidencia una relación directa entre la no correlación diagnóstica ecográfica/histopatológica de la corionicidad y estas condiciones, relación supuesta, pero no establecida de forma numérica hasta el momento de este estudio. (3).

Además se ha estimado que la sensibilidad y especificada en el diagnóstico de la corionicidad, es de cerca el $99 \%$ si esta se realiza de forma temprana $(2,7,13)$, por lo tanto es de esperar que a mayor edad gestacional de toma de la ecografía, menor sensibilidad y especificidad en el diagnóstico del tipo de corionicidad, por ende mayor riesgo de no diagnóstico preciso de la misma, lo cual expondría a nuestras pacientes, a seguimiento médico inadecuado, y a la no detección de complicaciones, tanto aquellas derivadas de la corionicidad como aquellas secundarias al embarazo gemelar; complicaciones con repercusión fetal y neonatal.

Tenemos que el 33\% de los embarazos gemelares monocoriales presentaron complicaciones derivadas de la corionicidad, siendo un 55\% las correspondientes a RCIU, prevalencia más elevada que la reportada en la literatura $(35 \%)$ en embarazos gemelares monocoriales. $(1,8)$

En nuestra población encontramos, que la mayoría de los casos de embarazos gemelares monocoriales, provenían del área metropolitana, con una media de edad materna de 28 años 
y que solo el 33\% presentaba básica secundaria o estudios superiores; además se encontró que el $20 \%$ no tenía ningún control prenatal y cerca del $31 \%$ contaban con máximo 3 controles prenatales, lo que indica que el $50 \%$ de nuestras pacientes no contaban con control prenatal adecuado, sin embargo es de tener en cuenta, las barreras en el acceso al control prenatal que se presentan en la población, el bajo nivel educativo de nuestra pacientes y el ser población de bajos recurso ,pudiera propiciar dicha condición.

La media de edad gestacional de finalización de la gestación fue de 34.3 semanas, y la causa más frecuente de término de la gestación en nuestra población fue trabajo de parto pretérmino y ruptura prematura de membranas en cerca de un $47 \%$ de los casos, lo que corresponde a literatura actual, dado que se reporta que en embarazos gemelares $<37$ semanas, el riesgo de presentar parto pretérmino es alrededor del $50 \%$ (4).

Se debe tener en cuenta, el hecho de ser un estudio retrospectivo, los datos recogidos corresponden a lo encontrado en la historia clínica, por lo que están sujetos a errores de interpretación, mala digitación y a ausencia de variables en algunos casos, resultado de su no referencia en la misma; sin embargo se revisó la base de datos de patología en cuanto de embarazos monocoriales se refiere y el diagnóstico histo/patológico fue nuestro gold estándar; además se logró obtener un tamaño de muestra adecuado y se analizaron variables de desenlace de mortalidad y morbilidad tanto fetal como neonatal.

\section{CONCLUSIÓN}

Teniendo en cuenta el porcentaje de no correlación diagnostica de la corionicidad en los embarazos gemelares monocoriales, la carga tanto de morbilidad como mortalidad que presentan, la relación directa entre la no correlación diagnostica en la ecografía realizada mayor de 20 semanas de gestación, con la presentación de resultado perinatal adverso fatal y la presentación de complicación neonatal, se considera importante el garantizar que la paciente con embarazo gemelar, específicamente el embarazo gemelar monocorial, tenga acceso a control prenatal de forma temprana, además valoración frecuente por el obstetra y 
el especialista en medicina materno fetal, dado el alto porcentaje de complicaciones que se pueden presentar; además tener acceso a realización ecografía temprana (I trimestre), para determinar el tipo de corionicidad de forma oportuna tal como lo estipulan las guías de práctica clínica.

Se recomienda además continuo entrenamiento y actualización por parte del personal encargado de realizar el estudio ecográfico inicial, para así establecer con confiabilidad el tipo de corionicidad, y poder realizar un seguimiento adecuado a la paciente con embarazo gemelar.

\section{BIBLIOGRAFÍA}

1. Becerra-mojica CH, Díaz-martínez LA, Contreras-garcía GA, Beltrán-avendaño MA, Salazar-martínez HA, Gutiérrez-sánchez LÁ, et al. HOSPITAL UNIVERSITARIO DE SANTANDER, Maternal and foetal outcomes in twin pregnancies seen at Hospital Universitario de Santander, A cohort study. 2015;66(1):37-45.

2. Births M, The IN. Multiple gestation associated with infertility therapy: An American Society for Reproductive Medicine Practice Committee opinion. Fertil Steril [Internet]. American Society for Reproductive Medicine; 2012;97(4):825-34.

3. Blumenfeld YJ, Momirova V, Rouse DJ, Caritis SN, Sciscione a., Peaceman a. M, et al. Accuracy of Sonographic Chorionicity Classification in Twin Gestations. J Ultrasound Med. 2014;33(12):2187-92.

4. Carroll SGM, Soothill PW, Abdel-Fattah S a, Porter H, Montague I, Kyle PM. Prediction of chorionicity in twin pregnancies at 10-14 weeks of gestation. BJOG. 2002;109(2):182-6. 
5. Devlieger RG, Demeyere T, Deprest J a, Van Schoubroeck D, Witters I, Timmerman $\mathrm{D}$, et al. Ultrasound determination of chorionicity in twin pregnancy: accuracy and operator experience. Twin Res. 2001;4(4):223-6.

6. Dias T, Arcangeli T, Bhide a, Napolitano R, Mahsud-Dornan S, Thilaganathan B, et al. First-trimester ultrasound determination of chorionicity in twin pregnancy. Ultrasound Obstet Gynecol. 2011;38(5):530-2.

7. Fa A, Negru A, Ae M, Grigoriu C, Re B, Șerban A. Special forms in twin pregnancy ACARDIAC TWIN / Twin reversed arterial perfusion ( TRAP ) sequence. 2015;8(4):517-22

8. Hoekstra C, Zhao ZZ, Lambalk CB, Dizygotic twinning.Hum Reprod Update. 2008;14(1):37

9. JM Martínez, E Gratacós, Clínica U, Múltiple DG, Fetal ÁDM, Materno-fetal SDM. Hospital clínic barcelona protocolo: gestación gemelar monocorial: retraso de crecimiento intrauterino selectivo (cirs). :1-4.

10. JM Martínez, E Gratacós, Clínica U, Múltiple DG, Fetal ÁDM, Materno-fetal SDM. Protocolo Gestación Gemelar Monocorial: Sindrome De Transfusión Feto-Fetal Severo ( Stff ). 2011;1-8.

11. Morin L, Lim K, Bly S, Butt K, Cargill YM, Davies G, et al. Ultrasound in Twin Pregnancies. Int J Gynecol Obstet. 2011;115(1):117-8.

12. Oldenburg a, Rode L, Bødker B, Ersbak V, Holmskov a, Jørgensen FS, et al. Influence of chorionicity on perinatal outcome in a large cohort of Danish twin pregnancies. Ultrasound Obstet Gynecol. 2012;39(1):69-74.

13. Shetty a., Smith a. PM. The sonographic diagnosis of chorionicity. Prenat Diagn. 2005;25(9):735-9. 


\section{JOSHA}

Journal of Science, Humanities and Arts

14. Stenhouse E, Hardwick C, Maharaj S, Webb J, Kelly T, Mackenzie FM. Chorionicity determination in twin pregnancies: how accurate are we? Ultrasound Obstet Gynecol. 2002;19(4):350-2. 